International Research Journal of Science and Technology Vol. 1, Issue.1 (2019) 6-12

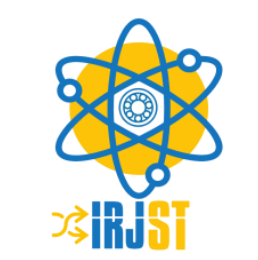

\author{
Available online at https://www.irjst.com/ \\ International Research Journal of Science and Technology \\ ISSN:2707-3955 \\ DOI: https://doi.org/10.46378/irjst.2019.010102
}

\title{
Review on Asymmetric Transformations with Chiral Phase-Transfer Catalysts Tesfaye Tebeka $^{{ }^{*}}$ and Atitegebe Abera ${ }^{2}$ \\ ${ }^{1,2}$ Department of Chemistry, Arba Minch University, Arba Minch, Ethiopia
}

Published: 2019

\begin{abstract}
This review focuses onasymmetric transformations with Chiral Phase-Transfer Catalysts and its application. Phase-transfer catalysis is practical methodology for organic synthesis. It is possible to achieve highly enantio selective transformations under phase-transfer conditions for a variety of $-\mathrm{C}-\mathrm{C}-$ $-\mathrm{C}-\mathrm{O}-$ and $-\mathrm{C}-\mathrm{N}-$ bond-forming reactions. The asymmetric transformations using modified cinchona alkaloids, chiral spiro ammonium salts and crown ether are among the primary source of effective chiral phase-transfer catalyst, which allows access to enantiomerically pure unnatural amino acids and synthetically useful adducts containing quaternary stereogenic centers. The advantage of this method is its simple experimental procedure, large chiral pool, mild reaction condition, inexpensive, environmentally benign reagent and use of simple and inexpensive reactants. Nowadays, it appears to be the most important synthetic method used in various fields of organic chemistry, and also found widespread industrial applications. This review summarizes the synthesis application, enantio selective transformation of some selected reaction, biological activities and catalytic activities of Phase-transfer catalysis and especial emphasis is given for organo catalysis. In asymmetric organo catalyst, it is possible to obtain chiral organic products in high enantio enriched form by steric hindrance approach method. The advantage of organic molecules as chiral catalysts complements the traditional organo-metallic and biological approaches to asymmetric catalysis.
\end{abstract}

Key Words

Chiral catalysis

Enantioselective transformation

Organocatalysis

Phase Transfer Catalysis

Steric hindrance

Copyright $\odot 2019$, Tesfaye Tebeka and Atitegebe Abera.This is an open access distribution, and reproduction in any medium, provided Access article distributed under the Creative Commons Attribution License the original work is properly cited License, which permits unrestricted use.

Citation: Tesfaye Tebeka and Atitegebe Abera. "Review on Asymmetric Transformations with Chiral Phase-Transfer Catalysts", International Research Journal of Science and Technology, 1 (1) 6-12,2019

\section{Introduction}

Heterogeneous chemical reactions between two reacting species located in immiscible phases are often inhibited due to the encounter problems. The crucial difficulty of bringing together a water soluble nucleophilic reagent and an organic water insoluble electrophilic reagent has been traditionally solved by the addition of solvent that is both water-like and organic-like.

\footnotetext{
${ }^{*}$ Corresponding author:Tesfaye Tebeka

] Department of Chemistry, Arbaminch University, Ethiopia e

Email: tesffish41@gmail.com
}

Alternatively, expensive dipolar aprotic solvents can be used, but they are suffering from the disadvantages of being difficult to separate from the reaction mixture during post reaction recoveryand expensive. Avery good method to overcome such problem is the uses of phasetransfer catalysts, which uses catalytic amounts of phase transfer agents and also facilitate the interphase transfer of specie that making reactions between reagents in two immiscible phases possible [1].

The phenomenon of rate enhancement of a reaction of between chemical species located in different phases by 
addition of a small quantity of an agent that extracts one of the reactants, most commonly an anion, across the interface into the other phase so that reaction can proceed via phase-transfer catalysis[1].

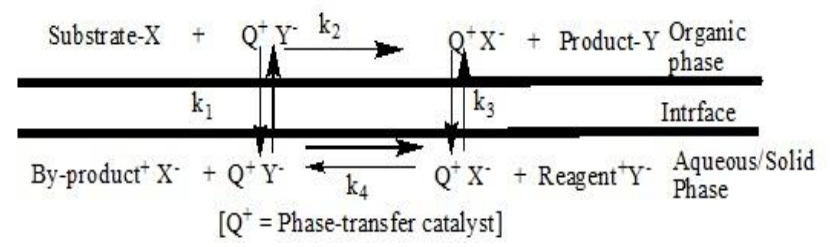

Figure1.Schematic representation of phase transfer catalysis

The phase transfer catalytic process involves at least three important steps (as depicted in Figure. 1). The reactant transfer step, in which the reactant anion is transferred from the aqueous or solid phase into the organic phase; phase transfer catalyst serves not only to transfer but also to activate the transferred anion by anion activation. Anion activation is related to the observed decrease of cation-anion interaction energy thereby lowering the free energy of activation for the displacement reaction that means if the free energy of the system is decreased so the rate of the reaction is accelerated [3]. Thereaction step in which the product is formed in some case at the interface and the product transfer step, in which the product anion is transferred back from the organic phase into the aqueous or solid phase. The efficiency of PTC is influenced by the bulkiness of the catalyst and its hydrophobicity and its counter ion [4].

Phase transfer catalysis reactions are highly attractive alternatives than the processes that use polar, watermiscible solvents. Because of these advantages, phasetransfer reactions have been recognized as "green" alternatives in to many homogeneous reaction processes, and they have found widespread application in synthetic organic chemistry [5-8].

Recently there are three general classification of asymmetric catalysis has been known. Organocatalysis, metal catalysis and enzymatic transformations, giving three fields that at the present time are complementary one to another, but in this review, more emphasis is given for organo asymmetric catalysis [1,9].Although there is no definite guidelines can be given to select the best catalyst for a given reaction system, analysis based on some of these factors can provide a suitable methodology to screen different phase transfer catalysts for a given system. Factors relevant in choosing a phase transfer catalyst are stability under reaction conditions, ease of preparation or availability of catalyst, ease of separation or recovery, activity and toxicity [1].

Two basic requirements of a Phase transfer catalyst (Starks et.al, 1978) such as: the first one is the PT agent must be cationic and must have enough organic structure to be able to partition the nucleophilic anion into the organic phase and the second one is the cation-anion bonding should be loose enough to ensure high anionic reactivity[10].This review, aimed to describe quaternary ammonium salts, crown ethers and the cinchona alkaloids with respect totheir ability to synthesis enantioenriched products.

\subsection{Mechanism of Phase Transfer Catalyst}

The principle of PTC is based on the ability of certain "phase-transfer agents" (the PT catalyst) to facilitate the transport of reagent from one phase into another (immiscible) phase where in the other reagent exists. Thus reaction is possible by bringing together the reagents which originally in different phases. However, it is also necessary that the transferred species is an active state for effective PT catalytic action, and that it is regenerated during the organic reaction [1].

The exact pathway for generating the reactive oniumcarbanion species remains the subject of controversy, however Starks extraction mechanism and the Makosza interfacial mechanism [11] are described below.

\subsubsection{Starks extraction mechanism}

In the Starks mechanism, the phase-transfer catalyst moves back and forth across the organic and aqueous phases. The onium salt equilibrates with the inorganic base in the aqueous phase, and extracts hydroxide into the organic phase. The onium hydroxide taken up hydrogen from the acidic organic compound to gives the reactive intermediate $\mathrm{Q}^{+} \mathrm{R}^{-}$(Fig.2) [11].

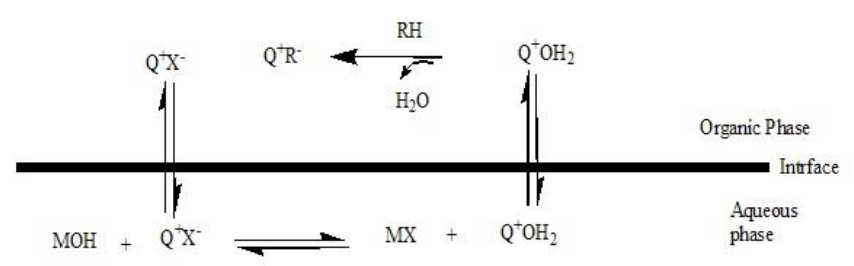

Figure 2. Starks extraction mechanism

\subsubsection{Makosza Interfacial Mechanism}

The advocated pathway of the interfacial mechanism is the first formation of metal carbanion at the interface of organic and aqueous phase in the absence of phase 
transfer catalyst, followed by the extraction of the formed metal carbanion species from the interface into the organic phase by the action of phase-transfer catalyst (Figure 3). Since asymmetric phase-transfer catalysts normally contain highly lipophilic chiral organic frameworks, and are reluctant to enter the aqueous phase, the Makosza interfacial mechanism seems plausible [11].

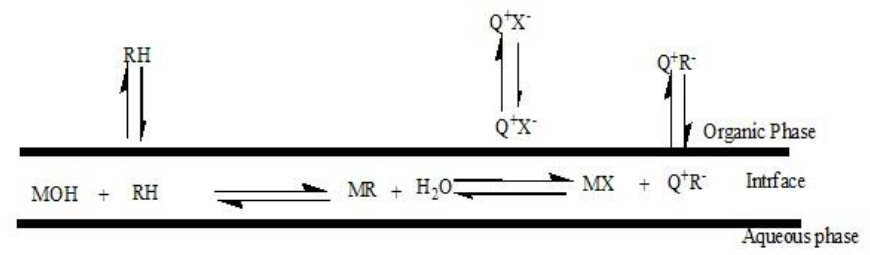

Figure 3.Makosza interfacial mechanism

\section{Asymmetric Organocatalysis}

The term 'asymmetric organocatalysis 'describes the acceleration of chemical reactionsthrough the addition of a catalytic amount of a chiral organic compound. In asymmetric organocatalyst, it is possible to obtain chiral organic products in enantioenrichedform. The use of solely organic molecules as chiral catalysts complements the traditional organometallic and biological approaches to asymmetric catalysis [9]. Recently, there has been a significant advance in chiral phase-transfer catalysis (CPTC) has been made. The cinchona alkaloids having pseudoenantiomeric forms and cinchonidinechiralspiroammonium salts and crown ether are among the primary source of effective chiral phasetransfer catalyst $[9,12]$.

\subsection{Cinchone alkaloids}

The alkaloids are available as pseudoenantiomers (Figure (4) that can achieve similar levels of asymmetric induction with opposite senses of enantioselectivity. If basic conditions are employed in PTC, an interfacial mechanism is implicated for sufficiently lipophilic quaternary catalyst. The quaternary ammonium cation transfers the anionic organic nucleophile into the organic phase. Where it can reacts with the organic electrophile $[13,14]$.

The chiral quinuclidinic nitrogen is the most important as it is responsible for the direct transfer of chirality in catalysis (Figure.4). The N-C (8) C (9)-O is usually the center of the catalytic activity.

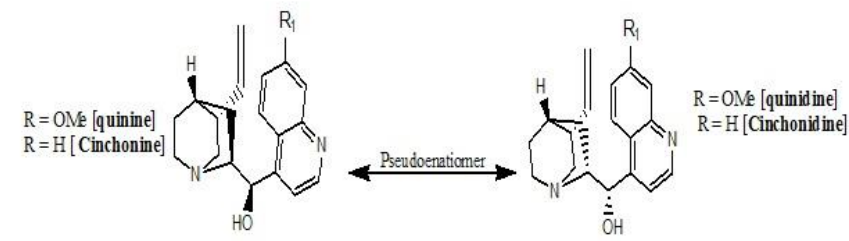

Figure 4.Cinchone alkaloids

Due to the close proximity of the catalyst, stereodifferentiation can be induced, selectively leading to the formation of a particular enantiomer/diastereoisomer, depending on the nature of the nucleophile/electrophile. The hydroxyl group at a carbon $\beta$ to the quaternary ammonium salt is used as an auxiliary that enhance anion-cation interaction via hydrogen bonding. Simple non-functionalized chiral ammonium salts are not sufficient to promote enantioselestivity. Multipoint interaction between the catalyst and the substrate is necessary $[15,16]$.

\subsection{Chiral quaternaryammonium Cations}

The chiral quaternaryammonium salt which is a powerful method in organocatalysis based on ion pair interactions between a nucleophilic anion and a positively charged catalyst. N-Benzyl quininium bromide is prepared very easily by mixing quinine and benzyl bromide in benzene-ethanol at room temperature for 26 hours (Figure 5) [17].

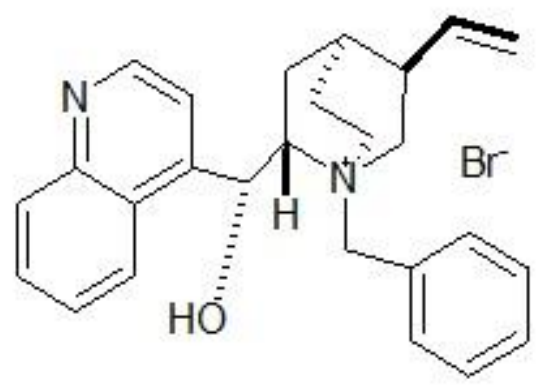

Figure 5. N-Benzyl quininium bromide

Avery good yield is obtained by the modification of Chiral PTC using N-[p-(trifluoromethyl) benzyl] cinchoninium bromide as the phase transfer catalyst (Figure 6a). Because the quinolone ring, the $\mathrm{C} 9-\mathrm{O}$ bond and the benzyl group all lie in a single plane and provide a multipoint interaction with the substrate by H-bonding. High enatioselective product is also obtained by $\mathrm{N}$ (trifluorobenzyl) cinchoninium bromide (Figure 6b), this is because the influence of hydrogen bonding in catalysts (Figure 6c) [18, 19]. 


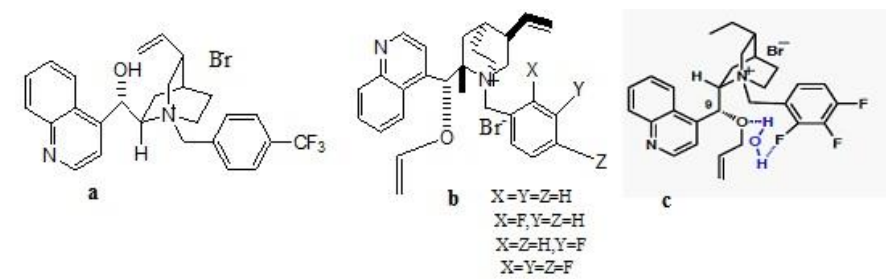

Figure 6. N-[p-(trifluoromethyl) benzyl] and N(trifluorobenzyl) cinchoninium derived phase transfer catalysts

The catalyst prepared from quinine and 9chloromethylanthracene showed enhanced enantioselectivity relative to the benzyl and $\mathrm{N}$-[p(trifluoromethyl) derivative (Figure 7), even a more enatimeric excess product is obtained when O-protected catalyst is used by addition ofsteric bulk substituent on the nitrogen atom (steric approach). This is because one face is inherently blocked by the bicyclic ring structure and another is blocked by alkylation of the oxygen on C9 and the remaining face is hindered bythe bulky anthracenylmethyl. The additional steric bulk whichisprovided by the anthracenylmethyl group should enhance the enantio-selectivity (19).

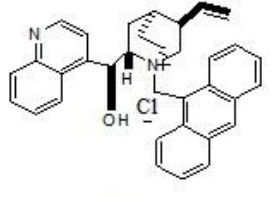

a

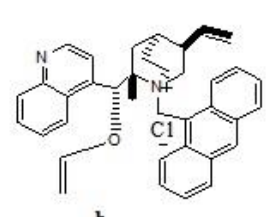

b

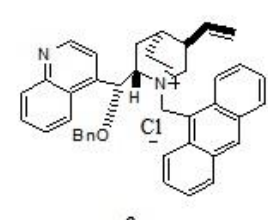

c
Figure 7. 9-chloromethylanthracene quinine derived catalyst

\subsection{Chiral Spiro ammonium Salts}

A more recently developed class of another organocatalysis uses a phosphoric acid moiety combined with chiral binaphtyl systems to mediate organic reactions. Highenantioselective product is obtained by Quaternary ammonium catalysts with new C2Symmetric chiral ammonium salts in the formation of $\alpha$ amino acids with only 1 mole\% catalyst loading (Figure 8) and the catalysts possess enhanced stability due in large part to the lack of a $\beta$-hydrogen that could promote Hofmann elimination [20,21].

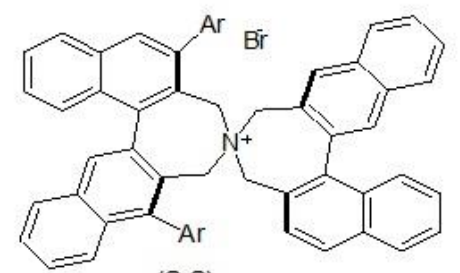

$\mathrm{Ar}=$

(S.S)

Figure $8 . \mathrm{C}_{2}$-symmetric chiral phase-transfer catalyst

\subsection{Crown ether}

Crown ether (Figure 9a) is the other important classes of phase-transfer catalysts which critically differs from the onium salt, in that the whole inorganic salt is transferred into the organic phasebycomplexing the cation in the cavity of the crown ether, and make the nucleophile susceptible to reaction (Figure 9b) [11,21, 22].Because their positive centers are well shielded from any interaction with anions by the cavity of the crown ether, the rates of $S_{N} 2$ reactions generally are vastly increased so as to synthesis high enantioenriched products.

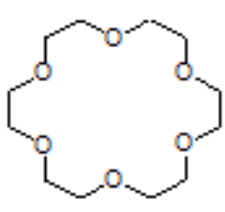

a

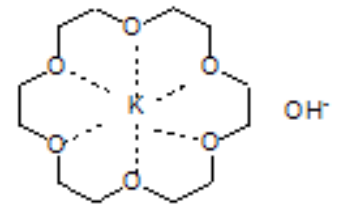

b
Figure 9.Crown ether (a) and action of crown ether (b)

\subsection{Synthetic application of Asymmetric phase- transfer catalysis}

PTC finds applications in a variety of reactions. Primary applications are in nucleophilicsubstitution reactions and in reactions in the presence of bases involving the deprotonation of moderately and weakly acidic organic compounds. Reactions carried out using PTC include oxidations, reductions, polymerizations, transition metal co-catalyzed reactions, synthesis of carbenes and further reactions of carbenes, addition reactions, condensations, and so on, which are often part of a multistep synthesis process for fine chemicals manufacture (1).In this review, asymmetric alkylation of active methylene, asymmetric epoxidation and aldol reactions are described below as an example.

\subsection{Alkylation:Synthesis of (R)-indacrinone}

Asymmetric alkylation of active methylene or methane compounds with alkyl halides, in the presence of asymmetric phase transfer catalysis, gives enatoselective products. The asymmetric alkylation of cyclic ketones in the presence of the Cinchona alkaloid ammonium salt derivative in $10 \mathrm{~mol} \%$ loading is possible to obtainindacrinone(biologically active compound for the protection of undesired side effect of uric acid retention) in quantitative yield of $95 \%, 92 \%$ at $25^{\circ} \mathrm{C}$ [24].

The high enantiomeric excess for the result is due to the electron withdrawing groups at the Para position of the benzyl ring and the tight ion pair intermediate (Figure10), formed through hydrogen bonding as well as 
electrostatic and $\pi-\pi$ stacking interactions[24].The mechanistic and kinetic studies for this reaction is explained as deprotonation of the substrate at the interface and enolate anion formation, anion extraction into organic phase and ion exchange to form the catalyst/substrate ion pair and chiral alkylation in the organic phase [24,25].

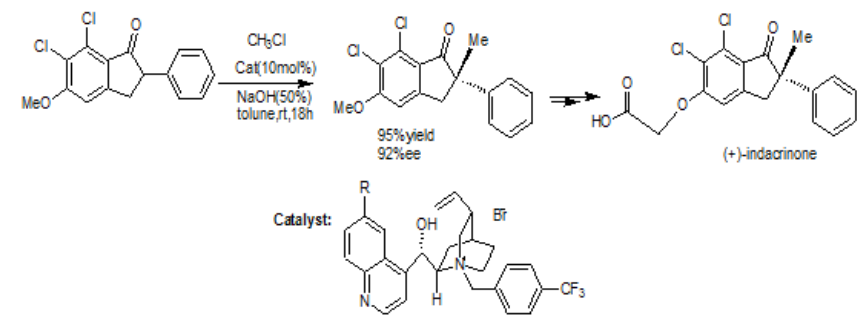

Figure 10. Asymmetric alkylation of cyclic ketones

\subsection{Asymmetric Alkylation: Amino Acid Synthesis}

For the asymmetric synthesis of $\alpha$-amino acids by O'Donnell et al., who used glycinate Schiff base as a key substrate [26].The asymmetric alkylation of glycinate Schiff proceeded smoothly under mild phase-transfer conditions, with $\mathrm{N}$-(benzyl) cinchoniniumchloride (Figure 11c) as a catalyst, to give the alkylation product (R) in good yield and moderate enantio-selectivity[24].

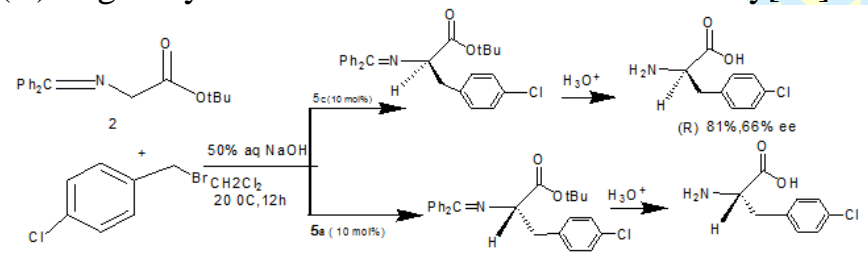

Figure11.Asymmetric synthesis of $\alpha$-amino acids

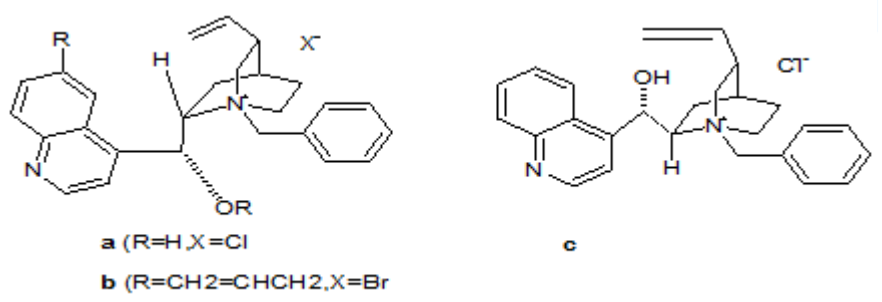

Figure 11a. N-(benzyl) cinchoninium derived catalyst

By simply switching to the cinchonidine-derived catalyst $6 \mathrm{a}$, the product could be obtained with the opposite absolute configuration (S) but with a similar degree of enantioselectivity. Further optimization with the hydroxyl-protected catalyst6b (second-generation catalyst) enhanced the enantioselectivity to $81 \%$ ee (27).

An important aspect of this reaction is the selective formation of the monoalkylatedproduct, without concomitant production of the undesired dialkylated product. This effect results from the much lower acidity of the remaining proton of the productascompared

totheprotonofglycinateSchiffandblockage of the second alkylation by the benzophenone imines. This reduced acidity and is also crucial for securing the configurationally stability of the newly formedstereogenic center under the reaction conditions [28].

Asymmetric phase-transfer alkylation of theglycinate Schiff basecan be achieved by using chiral phasetransfercatalysts derived from cinchona alkaloidbearing an $\mathrm{N}$-anthracenylmethyl group(thirdgeneration catalyst) toynthesize $\alpha$-amino acids with much higher enantio-selectivity [24].

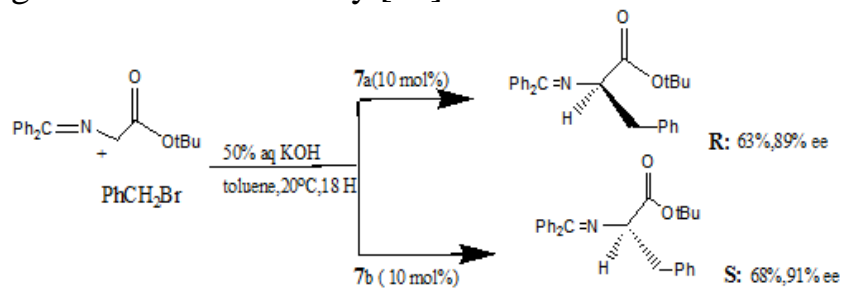

Figure 12. Synthesis of $\alpha$-amino acid by Nanthracenylmethylammonium salts

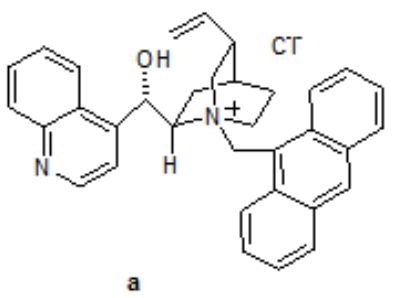

Figure13.Chiral phasetransfercatalysts bearing an $\mathrm{N}$-anthracenyl methyl

\subsection{Asymmetric Alkylation: L-dopa Synthesis}

A drug used for the treatment of Parkinson disease which is caused by degeneration of dopamine producing brain cells. Dopa is chiral, and only $(S)$-dopa(known as L-dopa) is effective in restoring nerve function [30].Typically only one enantiomer of a drug is biologically active. Only one stereoisomer of a drug molecule may fit perfectly in a chiral protein receptor, while the other may exhibit much less activity because of its inability to fit in that receptor. If we use a drug in the form of the racemate, serve a mixture of drugs. Racemic mixtures can also be considered compounds that contain 50\% impurity. Enantiomers can behave very differently in biological systems because they are different in chiral reagents, chemical and physical properties. 


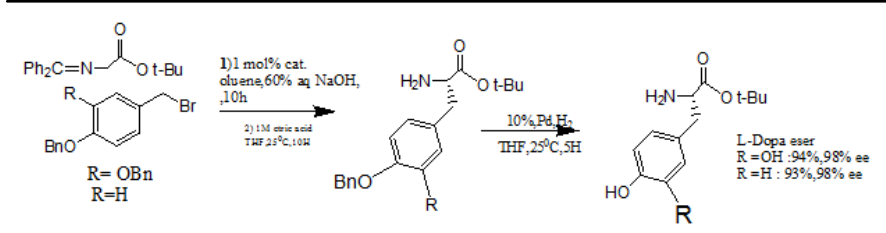

Figure 14.Asymmetric synthesis of L-dopa

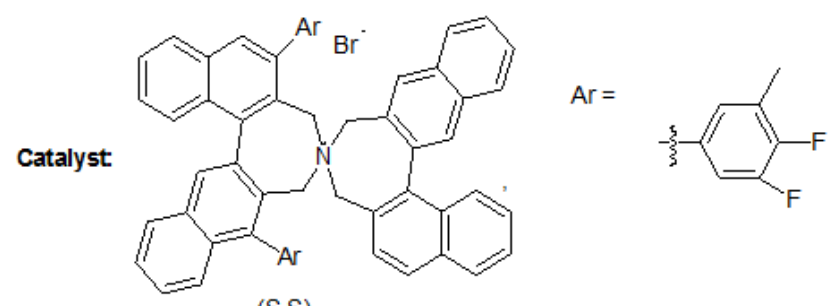

(S.S)

Figure $15 . \mathrm{C}_{2}$-symmetric chiral phase-transfer catalyst

\section{Conclusion}

Phase-transfer catalyst is practical methodology for organic synthesis. It is possible to achieve highly enantioselective transformations under phase-transfer conditions for a variety of $\mathrm{C}-\mathrm{C}, \mathrm{C}-\mathrm{O}$ and $\mathrm{C}-\mathrm{N}$ bondforming reactions. The asymmetric alkylation of cyclic and acyclic enolates using modified cinchona alkaloids allows access to enantiomerically pure unnatural amino acids and synthetically useful adducts containing quaternary stereogenic centers.

The advantage of this method is its simple experimental procedure, mild reaction condition, inexpensive and environmentally benign reagent. It is predicted that billions of people, particularly those in developing countries, face shortages of water and food and greater risks to health and life as a result of climate change. Climate change is anticipated to have far reaching effects on the sustainable development of developing countries. Alternative way to reduce such impact is make use of an eco-friendly productin all sectors. Use of simple and inexpensive reactants such as: $\mathrm{NaOH}, \mathrm{KOH}$, $\mathrm{K}_{2} \mathrm{CO}_{3}$ etc. instead of $\mathrm{NaH}$, KHMDS t-BuOK, etc.).Nowadays, it appears to be the most important synthetic method used in various fields of organic chemistry, and has also found widespread industrial applications.

In conclusion, Organocatalysis has several significant advantages over conventional metal catalysis. For example, there is usually fewer toxicity issues associated with organocatalysis. Of particular importance is that most reactions are tolerant of water and air, and are often easy to perform. These factors often affect metal catalyzedreactions, and that provides a significant advantage in terms of operational simplicity. Numerouscomprehensive publications are available that give a full account of the organocatalysis area [34-37].

Asymmetric phase-transfer alkylation of the glycinate Schiff baseof high enantio-pure productscan be achieved by using chiral phase transfer catalysts derived from cinchona alkaloid bearing an $\mathrm{N}$-anthracenylmethyl group (third-generation catalyst) to synthesize $\alpha$-amino acids with much higher enantioselectivity. Even higher enantioselectivity product can be achieved by using chiral $\mathrm{N}$-spiroammonium salts (fourth-generation catalyst) phase transfer catalysts.

The development of asymmetric phase transfer catalysis based on the use of structurally well-defined chiral, nonracemic catalysts is a great deal for the medical sector. This is because, only one stereoisomer of a drug molecule may fit perfectly in a chiral protein receptor, while the other may exhibit much less activity because of its inability to fit in that receptor.

The author confirms that this article content has no conflict of interest.

\section{References}

[1] Naik S.D. and Duraisamy L. K, AICHE Journal, 44 (1998)3.

[2] Starks C.M. \&Liotta C.L Phase-Transfer Catalysis Principles and Techniques, Academic, New York, 1978

[3] Lygo.B, Acc. Chem. Res., 37 (2004)518.

[4] D.Myers,3rd, 2006 John Wiley \&Sons,inc. Published Online: 2 NOV 2005; DOI: $10.1002 / 047174607 X . c h 1$

[5] Makosza M, Pure Appl. Chem., 72(2000)1399.

[6] M. J. O'Donnell, Asymmetric Phase-Transfer Reactions, Catalytic Asymmetric Synthesis, $2^{\text {nd }} e d$.

[7] Y.Sasson, R. Neumann, Handbook of PhaseTransfer Catalysis Eds., Blackie, London, 1997.

[8] M. J. O'Donnell, Phases-The Sachem Phase Transfer Catalysis Review 4(1998)

[9] Houk K. N. and List B, Eds. Acc. Chem. Res. 2004, 37, Nr 1-8.

[10] Takuya Hashimoto and KeijiMaruoka. The Basic Principle of Phase-Transfer catalysis. 
[11] Catalysis and Some Mechanistic Aspects Asymmetric Phase Transfer Catalysis. 2008 WILEY-VCH GmbH \& Co. KGaA, WeinheimISBN: 978-3-527-31842-1.

[12] Rahul Palchaudhuri "Chiral phase-transfer catalysis (CPTC) by modified cinchona alkaloids", March 12, 2007.

[13] Bolm,C.,Schiffers I., Atodiresei, I., Ozcubukcu, S., Raabe G.,"Asymmetric Anhydride Opening: Optimization and Applications Master of Science thesis submitted to Institute of Organic Chemistry" RWTH-Aachen University, Oct,2001-March 2005.

[14] MaruokaK.andOoiT., Chem. Rev., 103(2003)301.

[15] Martin J. O'Donnell, Acc. Chem. Res, 37 (8), (2004)506.

[16] Hartmuth C. Kolb, Michael S. Van Nieuwenhze, Chem. Rev,94 (8) (1994)2483.

[17] Katrien B.\&Jacobsen Eric N, AngewChemInt Ed Engl, 52(2) (2013)534.

[18] Y. Sasson, Ronny Neumann Handbook of Phase Transfer Catalysis, page 111.

Website: http://www.springer.com/ascessed online on $19 / 62015$.

[19] Darrell Crick and Marc Halpern. PTC Commun.Inc. 17 (2003).

[20] Ooi, T., Kameda, M. \&Maruoka, K. J. Am. Chem. Soc. 121 (1999)6519.

[21] MaruokaK,Pure Appl. Chem., 77(2005)1285.

[22] Maruoka K, Angew. Chem. Int. Ed., 44(2005)625.

[23] Maruoka K. \& T. Ooi, Angew. Chem. Int. Ed, 46 (2007)4222.

[24] O'Donnell M., "Asymmetric Phase-Transfer Catalysis",Catalytic Asymmetric Synthesis,Ojima ed., Wiley-VCH, 1993.

[25] O'Donnell, M. J. 2001. Ethyl N(Diphenylmethylene)glycinate. e-EROS Encyclopedia of Reagents for Organic Synthesis.

[26] A.Esikova, T. S. Nahreini, M. J. O Donnell in Phase-Transfer Catalysis (Ed.: M. E. Halpern), American Chemical Society,Washington, DC, 1997, chap. 7 (ACS Symposium Series 659).

[27] Martin J. O'Donnell.Academic. Chem. Res, 37 (8) (2004) 506.

[28] Takashi Ooi, Minoru Kameda, and KeijiMaruoka, J.Am. Chem. Soc., 125(2003)5139.
[29] Gasparski C. M. \& Miller M. J, Tetrahedron, 47(1991)367.

[30] Ooi T., Kameda M., Taniguchi \&Maruoka K, J. Am.Chem.Soc, 126 (2004)9685.

[31] Dalko P.I. and Moisan L, Angew. Chem. Int. Ed. Engl, 43 (2004)5138.

[32] Dalko P.I. \&Moisan L, Chem. Int. Ed. Engl, 41(2001) 3726.

[33] List B., Adv. Synth. and Cat., 346(2004) 1021.

[34] List B, Asymmetric organocatalysis. Chem. Commun, (2005) 719. 